\title{
Regular paracetamol in severe dengue: a lethal combination?
}

Chin Seng $\underline{G a n}^{1}$, MBBS, MPaeds, Sze Yee Chong${ }^{1}$, MD, MPaeds, Lucy Chai See $\underline{L u m}^{1}$, MBBS, MRCP, Way Seah $\underline{\operatorname{Le}}^{1}, \mathrm{MD}, \mathrm{FRCPH}$

ABSTRACT An eight-month-old female infant with severe dengue disease, who was repeatedly given therapeutic paracetamol for severe dengue, developed fulminant liver failure with encephalopathy, gastrointestinal haemorrhage and severe coagulopathy. She responded to supportive measures and $\mathrm{N}$-acetylcysteine infusion. This case highlights the potential danger of administering repeated therapeutic doses of paracetamol in childhood severe dengue disease with hepatitis.

Keywords: hepatitis, paracetamol, severe dengue

Singapore Med J 2013; 54(2): e35-e37

\section{INTRODUCTION}

Dengue fever (DF) is a global health problem. ${ }^{(1)}$ While the main manifestation in severe dengue disease (SDD) is primarily haemorrhage, dengue hepatitis is an increasingly well-known complication of SDD. ${ }^{(2-4)}$ In severe hepatitis, fulminant hepatic failure (FHF) has been reported. ${ }^{(5-6)}$ The new World Health Organization (WHO) guidelines for DF recommend the administration of paracetamol (PCM) for high fever if the patient is uncomfortable. ${ }^{(7)}$ However, these guidelines do not specify whether PCM should be given in the presence of hepatitis. ${ }^{(7)}$

We report the case of an eight-month-old infant with SDD who was given therapeutic doses of PCM for fever. The illness was complicated by FHF. This case highlights the risk of severe liver injury in children with acute dengue hepatitis who are given repeated therapeutic doses of PCM.

\section{CASE REPORT}

An eight-month-old female infant was referred for further management of SDD, encephalopathy, coagulopathy and hepatitis. Her fever had started 13 days ago. She was admitted to a local hospital on Day 4 of the illness with a temperature of $39.0^{\circ} \mathrm{C}$ and a generalised maculopapular rash. There was no history of use of herbs or other traditional medicine. There was also no other signs that would suggest the presence of other concomitant infections. On admission, her haemoglobin level was $62 \mathrm{~g} / \mathrm{L}$, white blood cells was $7.4 \times 10^{9} / \mathrm{L}$ and platelet count was $33 \times 10^{9} / \mathrm{L}$. Dengue NS1 antigen and dengue serology immunoglobulin M were both positive. The patient developed an episode of generalised seizures soon after admission. Intravenous fluids were started and her condition improved. Despite the decrease in haematocrit level (48\% on Day 5 of illness, 33\% on Day 6 , $31 \%$ on Day 7 ), the patient received intravenous fluids at $4 \mathrm{~mL} / \mathrm{kg} / \mathrm{hr}(0.45 \%$ saline, $5 \%$ dextrose solutions).
On Day 11 of illness, the patient had haematemesis. She was jaundiced and had fluid overload. Her liver enzymes were markedly raised, with aspartate transferase (AST) at 1,539 IU/L and alanine transferase (ALT) at 5,497 IU/L. There was no liver function test done before this. The international normalised ratio (INR) was 2.89 and prothrombin time (PT) was $26.4 \mathrm{~s}$. She was referred to the paediatric intensive care unit of University Malaya Medical Centre for further management. A review of the patient's treatment at the referring hospital showed that she had been given regular PCM at a dose of $105 \mathrm{mg}$ (15 mg/kg/dose), four times a day for a total of seven days. On examination, the patient appeared ill, oedematous, jaundiced, pale and very drowsy. There was a generalised petechial rash, hepatosplenomegaly, gross ascites and bilateral basal crepitations over both lungs. Haematemesis and malaena were present. There was also generalised hypertonia and hyperreflexia of all four limbs (hepatic encephalopathy grade III). ${ }^{(8)}$ She was intubated and ventilated immediately. The diagnosis was post-dengue shock syndrome with FHF, possibly contributed by PCM overdose.

The patient's initial blood investigations showed total bilirubin $178 \mu \mathrm{mol} / \mathrm{L}$, conjugated bilirubin $111 \mu \mathrm{mol} / \mathrm{L}$, AST 2,684 IU/L, ALT $806 \mathrm{IU} / \mathrm{L}$, alkaline phosphatase $806 \mathrm{IU} / \mathrm{L}$, creatinine $26 \mu \mathrm{mol} / \mathrm{L}$ and serum ammonia $170.5 \mu \mathrm{mol} / \mathrm{L}$, with coagulopathy $(\mathrm{INR}>8, \mathrm{PT}>100 \mathrm{~s}$, activated partial thromboplastin time $76.8 \mathrm{~s}$, D-dimer test positive). Serum PCM level at 20 hours after the last dose was $0.1 \mathrm{mmol} / \mathrm{L}$ above the PCM treatment nomogram. ${ }^{(9)}$ The illness was complicated by hypoglycaemia-induced seizures and upper gastrointestinal bleeding. A computed tomographic examination of the brain showed no intracranial bleeding or cerebral oedema. The patient was given multiple transfusions of blood products and intravenous vitamin $\mathrm{K}$. Intravenous infusion of $\mathrm{N}$-acetylcysteine at a dose of $10 \mathrm{mg} / \mathrm{kg} / \mathrm{hr}$ was commenced. She was encephalopathic

${ }^{1}$ Department of Paediatrics, University Malaya Medical Centre, Kuala Lumpur, Malaysia 
for seven days. INR (> 8.0) and PT (> 100 s) were persistently deranged despite repeated transfusion of blood products. The subsequent course was also complicated by hospital-acquired infections. As emergency liver transplantation for FHF in young infants is not available in Malaysia, supportive measures were continued. On Day 15 of admission, the patient's coagulation profiles gradually improved. She was extubated on Day 18. Upon discharge from the hospital on Day 25, she still had residual left hemiparesis. At follow-up review two months after the initial illness, no residual neurological deficit was noted.

\section{DISCUSSION}

FHF due to PCM overdose may be due to either a single large overdose or cumulative, multiple overdoses. ${ }^{(10,11)}$ The latter has been increasingly recognised as an important cause of FHF due to PCM overdose. ${ }^{(11)}$ In a recent Australian and New Zealand guidelines for the management of PCM poisoning, a single dose of $200 \mathrm{mg} / \mathrm{kg}$ or more of PCM ingestion over a period of less than eight hours in children younger than six years old is considered likely to be associated with hepatic injury. ${ }^{(9)}$ For those with repeated supratherapeutic ingestion, the doses are considerably lower, depending on the duration of ingestion. ${ }^{(9)}$ However, the guidelines did not specify the dose that may possibly be associated with liver injury in children with pre-existing liver conditions. ${ }^{(9)}$ Mild to moderate hepatitis is common in dengue, with its occurrence reported in $71 \%$ of children with dengue. Severe hepatitis (serum ALT > $300 \mathrm{IU} / \mathrm{L}$ ) was noted in 15\%. ${ }^{(4)}$ The mortality rate of children with severe hepatitis in dengue was $67 \%{ }^{(4)}$ Hence, hepatic involvement should be suspected in all patients, not only in those with DF, but possibly also in those with other viral or fulminant bacterial infections. Liver functions should be determined early, and in cases of prolonged fever, at regular intervals.

Our patient had clinical evidence of severe dengue with supportive dengue serology. During the course of her illness, she was given repeated therapeutic doses of PCM for one week. Her serum PCM level was within the toxic range. Arguably, it is difficult to ascertain whether repeated administration of PCM in this patient ultimately resulted in $\mathrm{FHF}$, as both severe dengue and repeated PCM administration can lead to hepatic injury. There have not been many case reports on the effect of repeated PCM administration in therapeutic doses and its effect on liver function in patients with SDD, although some authors have advised checking for liver enzymes profile before administration of PCM in patients suspected of SDD. ${ }^{(12)}$ There have been evidences, however, both in vitro and in vivo, that the metabolism of PCM is reduced in patients with hepatitis. ${ }^{(13,14)}$ Although at present, there is no study on the metabolism of PCM in cases of dengue complicated by concomitant hepatitis, it is likely that the result may be significant. The new $\mathrm{WHO}$ guidelines on DF allows PCM to be prescribed to relieve discomfort from fever. ${ }^{(7)}$ However, there is no clear guideline on the regular use of PCM in DF complicated by dengue hepatitis. Caution should thus be exercised when giving repeated therapeutic doses of PCM in children with hepatitis, even in the absence of hepatic function impairment.

Since hepatitis is common in childhood dengue haemorrhagic fever, we suggest that screening for hepatic function derangement be done in all cases of SDD. In children with SDD and deranged liver enzyme levels, considerable care should be exercised when PCM is prescribed for the relief of fever. We suggest that therapeutic dosing should not always be at the maximum dose even though the recommended therapeutic dose of PCM is $10-15 \mathrm{mg} / \mathrm{kg}$ (of body weight) four times daily. Additionally, in the presence of hepatic involvement, clinicians should consider reducing the doses of drugs that are metabolised by the liver, including PCM. Dosing should be guided by the monitoring of the serum levels of the drug prescribed. At present, WHO guidelines do not encourage the use of other nonsteroidal antiinflammatory drugs, such as ibuprofen or antipyretics, in DF. In this aspect, WHO's guidelines on dengue should also be updated to clarify the indications, dosing levels, duration and a safe antipyretic alternative for children with DF.(7)

Although the prognosis of FHF secondary to PCM is generally quite favourable with a spontaneous recovery rate of $94 \%$, patients with grade III encephalopathy have a recovery rate of only $33 \% .^{(10)}$ In conclusion, in our report of a young infant with dengue haemorrhagic fever complicated by FHF, we suggest caution in the administration of repeated therapeutic doses of PCM in children with dengue or other medical conditions where the metabolism of PCM could be impaired.

\section{REFERENCES}

1. Guzman MG, Halstead SB, Artsob H, et al. Dengue: a continuing global threat. Nat Rev Microbiol 2010; 8(12 Suppl):S7-16.

2. Smith DR, Khakpoor A. Involvement of the liver in dengue infections. Dengue Bull 2009; 33:75-86.

3. Chongsrisawat $\mathrm{V}$, Hutagalung $\mathrm{Y}$, Poovorawan $\mathrm{Y}$. Liver function test results and outcomes in children with acute liver failure due to dengue infection. Southeast Asian J Trop Med Public Health 2009; 40:47-53.

4. Prakash O, Almas A, Jafri SM, et al. Severity of acute hepatitis and its outcome in patients with dengue fever in a tertiary care hospital Karachi, Pakistan (South Asia). BMC Gastroenterol 2010; 10:43.

5. Ling LM, Wilder-Smith A, Leo YS. Fulminant hepatitis in dengue haemorrhagic fever. J Clin Virol 2007; 38:265-8.

6. Vijayalakshmi AM, Devaprasath S. Fulminant hepatic failure in primary dengue infection. Indian Pediatr 2010; 47:280.

7. World Health Organization. Dengue Guidelines for Diagnosis, Prevention and Control. Available at: whqlibdoc.who.int/ publications/2009/9789241547871_eng.pdf. Accessed February 17, 2011.

8. Alonso EM, Squires RH, Whitington PF. Acute liver failure in children. In: Suchy FJ, Sokol RJ, Balistreri WF, eds: Liver Disease in Children, 3rd ed. New York: Cambridge University Press, 2007.

9. Daly FF, Fountain JS, Murray L, Graudins A, Buckley NA. Guidelines for the management of paracetamol poisoning in Australia and New Zealand. A consensus statement from clinical toxicologists consulting to the Australasian poisons information centres. Med J Aust 2008; 188:296-301.

10. Squires RH Jr, Shneider BL, Bucuvalas J, et al. Acute liver failure in children: the first 348 patients in the pediatric acute liver failure study group. J Pediatr 2006; 148:652-8.

11. Mahadevan SB, McKiernan PJ, Davies P, Kelly DA. Paracetamol induced hepatotoxicity. Arch Dis Child 2006; 91:598-603. 
12. Vazquez-Pichardo M, Rosales-Jimenez C, Rojas-Espinosa O, LopezMartinez I, Moreno-Altamirano MMB. Is liver damage dependent on the serotype of dengue virus? A study in Mexico. Dengue Bull 2006; 30:114-20.

13. Jorup-Ronstrom C, Beermann B, Wahlin-Boll E, Melander A, Britton S.
Reduction of paracetamol and aspirin metabolism during viral hepatitis. Clin Pharmacokinet 1986; 11:250-6.

14. Maddox JF, Amuzie CJ, Li M, et al. Bacterial- and viral-induced inflammation increases sensitivity to acetaminophen hepatotoxicity. J Toxicol Environ Health A 2010; 73:58-73. 